\title{
ALCOXISILANOS EN LA CONSOLIDACIÓN DE MATERIALES PÉTREOS
}

\author{
Isabel del Hierro ${ }^{1}$ y Yolanda Pérez ${ }^{1}$
}

\begin{abstract}
Resumen:
El proceso de polimerización inorgánico sol-gel utilizando monómeros del tipo alcoxisilanos como precursores se utiliza con éxito en el proceso de consolidación de materiales pétreos. Fórmulas comerciales con compuestos tetra y alquil trialcoxosilano como principal componentes se utilizan con éxito en la consolidación de la piedra. La baja viscosidad de estos compuestos les permite una elevada penetrabilidad en los poros de la piedra, donde polimerizan mediante un proceso clásico sol-gel formando estructuras muy fuertes con enlaces $\mathrm{Si}-\mathrm{O}$, similar a un vidrio amorfo de baja temperatura. En la actualidad se investigan nuevas formulas y nuevos procesos sol-gel para eliminar los dos principales problemas de este tipo de consolidantes, su tendencia a la fractura en los poros de la piedra y su incapacidad para consolidar rocas del tipo calcita.
\end{abstract}

\section{Palabras clave:}

Alcoxisilanos, consolidación, materiales pétreos.

\section{Abstract:}

The sol-gel process has been found to be successful in applications for the conservation of stonework. Commercial products containing alkoxysilanes, such as tetraethoxysilane (TEOS), are commonly used for the consolidation of stone. These products polymerize within the pores of stone by means of a classic sol-gel process, thereby strengthening the material. Their advantages are well-known: the low viscosity of TEOS allows it to penetrate deeply into porous stone; after polymerization, which occurs upon contact with environmental moisture, a stable gel with a silicon oxygen backbone is formed. New formulas and new sol-gel procedures are under research in order to overcome the main two well-known drawbacsk of these conservation products. Their tendency to form brittle gels that are highly susceptible to forming cracks inside the stone and their effectiveness on consolidate carbonate stones, as they can only bond mechanically by filling in the irregularities in the stone.

\section{Key words:}

Alcoxilians, consolidation, stone materials.

1 Departamento de Biología, Geología y Química Inorgánica. Escuela Superior de Ciencias Experimentales y Tecnología. C/Tulipán S/N 28934 Móstoles (Madrid). 
El crecimiento exponencial de compuestos químicos en el ambiente a lo largo del siglo XX ha acelerado la degradación del patrimonio cultural pétreo expuesto de forma permanente a un medio cada vez más hostil. Los procesos responsables de este deterioro se han estudiado en profundidad; destacando contaminación atmosférica, acción del agua, cristalización de sales y acción de organismos vivos.

En un intento de proteger y/o restaurar este patrimonio ya desde mediados del siglo XX se empezaron a utilizar diferentes tratamientos con el objetivo de consolidar la piedra, es decir, restablecer la cohesión entre las partículas de la piedra deteriorada física o químicamente.

El proceso de consolidación ideal debería pasivar la superficie del mineral frente al desgaste y/o la degradación atmosférica; debería mantener las propiedades de la piedra respecto al transporte de agua y aumentar su hidrofobicidad; de esta forma se podría minimizar la adsorción de agua y evitar los problemas asociados a los ciclos de hielo y deshielo y cristalización de sales. Un requisito adicional, pero no menos importante, de un proceso de consolidación o fortalecimiento ideal sería el del mantener la estética de la piedra

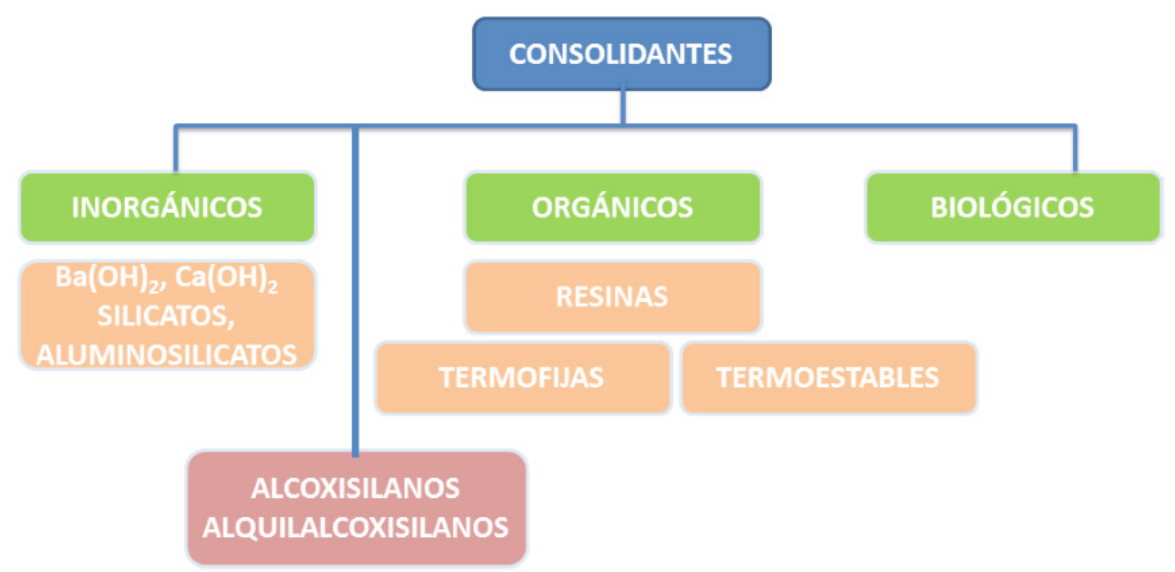

Fig. 1. Clasificación de Consolidantes para materiales pétreos.

El tratamiento debería ser, además, sencillo de aplicar y estable frente a la radiación ultravioleta. El compuesto utilizado debería presentar buenos niveles de penetrabilidad en los poros de la piedra y adherirse a la superficie de la misma.

Estos requisitos son muy difíciles de cumplir dada la heterogeneidad inherente de los materiales pétreos, diferente mineralogía, porosidad, tamaño de la superficie a tratar. Un sellado completo de la superficie taponaría los poros y dejaría agua entrapada en zonas próximas a la superficie, susceptible de sufrir ciclos de hielo y deshielo y capaz de producir delaminación de la superficie a tratar. Si el tratamiento es permeable al vapor de agua, pero no al agua líquida, se favorecería 
la deposición de sales a partir de la evaporación del agua en la disolución adsorbida. En resumen, no existe un consolidante universal, debe elegirse aquel que mejor se ajuste a las características específicas de la piedra².

Los materiales que tradicionalmente se han usado como consolidantes se clasifican en consolidantes clásicos inorgánicos y orgánicos y los Ilamados organosilícicos o alcoxisilanos. (Fig. 1)

Los tratamientos inorgánicos muy utilizados a final del siglo XIX, comienzos del XX suelen generar cambios cromáticos en la piedra y provocan la aparición de sales. Junto con su baja penetrabilidad y alta fragilidad, desaconsejan su uso. Este hecho, justificó la aparición en el mercado de productos basados en compuestos orgánicos junto a otros aditivos. Se desarrollaron las resinas termoplásticas, resinas vinílicas y acrílicas (polimetacrilato) o termoestables como las epoxídicas, poliéster, etc. Estas resinas han sido las más utilizadas desde mediados del siglo $X X$, aunque su uso se ha modificado con el paso del tiempo. Las resinas epoxídicas presentan excelentes cualidades adhesivas sin embargo sufren un rápido deterioro por acción de la radiación ultravioleta. El poliéster presenta también limitaciones, ya que suelen reducir la permeabilidad al vapor de agua, y sufre también un rápido envejecimiento con notables cambios cromáticos, fractura e incluso pulverización con el paso del tiempo.

Los monómeros alcoxisilano se pueden mezclar con agua y alcohol para preparar mezclas de baja viscosidad. Pueden aplicarse sobre la superficie con un cepillo o en spray y el compuesto es absorbido en los poros de la piedra por acción capilar, entonces comienza el proceso de gelificación. El polímero resultante tiene una estructura de enlaces Si-O, similar a un vidrio amorfo de baja temperatura, muy fuerte y con elevada estabilidad térmica y resistente la oxidación y a la radiación ultravioleta. Los grupos funcionales alcoxido enlazados al átomo central de silicio se pueden cambiar por grupos alquilo que no reaccionan y que aportan propiedades hidrófobas al producto final. Lo que resulta interesante en este tipo de compuestos es que, en teoría, durante el proceso de polimerización tienen la capacidad de reaccionar con el agua presente en la superficie de la piedra para formar enlaces $(\mathrm{Si}-\mathrm{O})_{n}$ con los grupos hidroxilo en la superficie de la misma.

Fue Berzelius en 1824 quien sintetizó por primera vez tetraclorosilano, $\mathrm{SiCl}_{4}$. Ebelman en 1846 y Ladenberg en 1874 sintetizaron nuevos compuestos de estas familia, tetraetoxisilano $\mathrm{Si}\left(\mathrm{OCH}_{2} \mathrm{CH}_{3}\right)_{4}$ (TEOS) y metiltrietoxisilano $\mathrm{SiCH}_{3}(\mathrm{O}-$ $\left.\mathrm{CH}_{2} \mathrm{CH}_{3}\right)_{3}$ (MTEOS). Aunque tuvieron que pasar casi cien años, antes de que estos compuestos empezaran a utilizarse como unidad fundamental y base de una nueva química, ya en 1861 Hoffmann sugirió la existencia de "éter silícico" una forma de (TEOS) en la fórmula utilizada para consolidar el parlamento de Londres.

2 (a)G. Wheeler. Alkoxysilanes and the consolidation of stone. Getty Publications. (2005). ISBN 978-0-89236-815-0. C. A. (b) C.A. Price. Stone Conservation. Getty Publications. (1996). ISBN 0-89236-389-4. 
El proceso de polimerización de estos monómeros líquidos conocido como sol-gel, es un proceso que transcurre a temperatura ambiente y en agua, conocido por utilizarse para preparar óxidos inorgánicos ${ }^{3}$ (Fig. 2). Los monómeros inorgánicos en disolución acuosa reaccionan para formar nanopartículas coloidales (el sol), con dimensiones entre $1 \mathrm{~nm}$ y $1 \mu \mathrm{m}$, y en una etapa posterior las partículas coloidales se agregan mediante la formación de enlaces y quedan en suspensión.
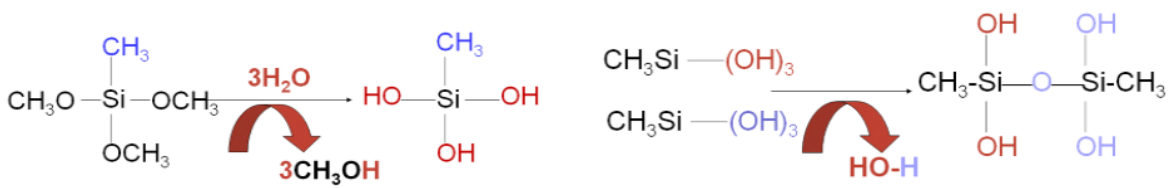

A

B

Fig. 2. Reacciones de (A) hidrólisis y (B) condensación de los monómeros inorgánicos.

Finalmente, sufren un proceso de gelificación por la formación de redes interconectadas, que están a su vez llenas de disolvente. Con el tiempo las partículas coloidales y las especies de sílice condensadas se unen para formar una red tridimensional. Las características físicas de esta red dependen del tamaño de las partículas y del grado de entrecruzamiento existente antes de la gelificación. Con la gelificación la viscosidad aumenta drásticamente y se obtiene un sólido que se ajusta al molde que lo contiene. Otras etapas de este proceso como son el envejecimiento, el secado o pérdida de disolvente y la densificación, o formación de una cerámica densa, condicionaran, finalmente, el proceso de consolidación.

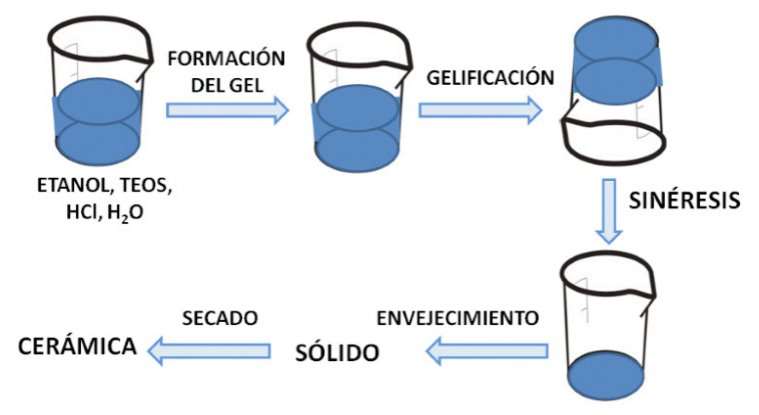

Fig. 3. Etapas del proceso sol-gel.

3 R. Corriu, N. Trong Anh. Molecular Chemistry of Sol-Gel Derived Nanomaterials. Wiley and sons (2009). 
Sorprendentemente, solo se han utilizado un número muy limitado de alcoxisilanos en los procesos de consolidación del patrimonio pétreo: Tetrametoxisilano (TMEOS), Metiltrimetoxisilano (MTMOS), Metiltrietoxisilano (MTEOS) y Tetraetoxisilano (TEOS). La explicación es que deben formar redes tridimensionales en el proceso de polimerización y, por tanto, deben tener al menos tres grupos reactivos enlazados al átomo central de silicio. Estos líquidos no son tóxicos dadas sus bajas presiones de vapor e igualmente generan alcoholes no tóxicos durante el proceso de polimerización. Su reactividad frente al agua es moderada, si reaccionasen demasiado rápido la capacidad de penetración del líquido en la piedra sería muy limitada. En la preparación de las fórmulas para utilizar como consolidantes se deben tener en cuenta otros factores como el disolvente a utilizar y el uso de catalizadores ácidos o básicos, ya que influirán de forma decisiva en las características finales de la cerámica final formada en los poros de la piedra, en última estancia responsable de la consolidación de las partículas pétreas (Fig. 4).

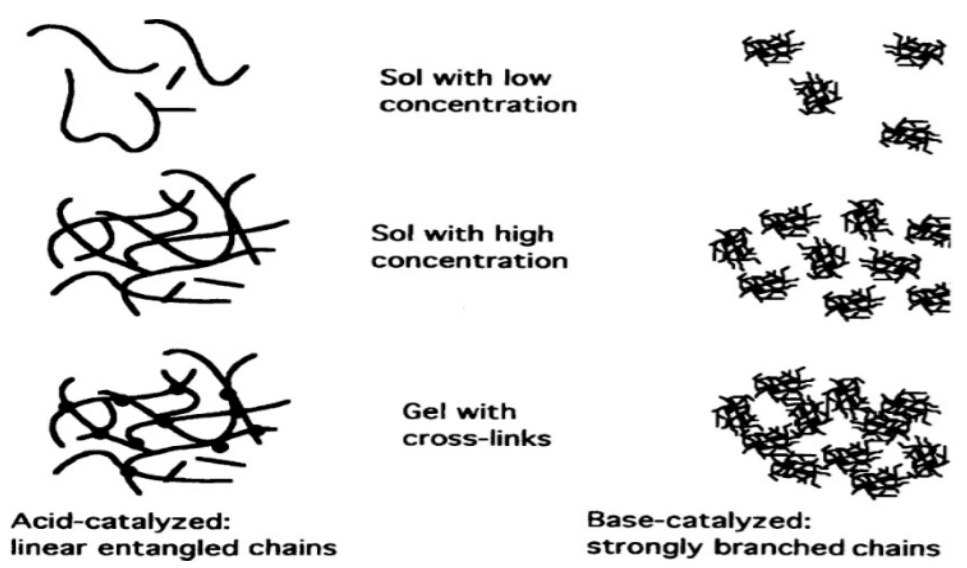

Fig. 4. Geles obtenidos mediante el uso de catalizadores ácidos y básicos.

Un factor muy importante es la naturaleza del medio a consolidar, debe tenerse en cuenta no solo a la hora de elegir el tratamiento sino también en el resultado final. Los alcoxisilanos no son eficaces en la consolidación de rocas carbonatadas (caliza y mármol) con alto contenido en carbonato de calcio $\left(\mathrm{CaCO}_{3}\right)$ ya que se evaporan rápidamente tras su aplicación. Son muy eficaces, sin embargo, para la consolidación de silicatos y rocas silíceas $\left(\mathrm{SiO}_{2}\right)$.

Las formulas comerciales utilizadas tradicionalmente son:

- Wacker $\mathrm{OH}$ (Patente de los años 70); fórmula basada en el uso de TEOS con metiletilcetona, acetona y etanol ( $25 \% \mathrm{v} / \mathrm{v})$ como disolvente y catalizada con dibutilestañodilaureato. 
- Wacker H (mezcla equimolar de MTMOS y TEOS).

- Wacker OH100 y H100 (sin disolvente).

- Conservare OH100 y H100 (USA).

- Keim's Silex OH y H (sin tolueno como disolvente).

- Tegokanov $\mathrm{V}$, fórmula basada en el uso de TEOS con $17 \% \mathrm{w} / \mathrm{w}$ nafta (mezcla de hidrocarburos arómaticos de bajo punto de ebullición) y etanol $(17 \% \mathrm{w} / \mathrm{w})$ y catalizada por dibultilestañodilaureato.

- Tegokanov T: (mezcla equimolar de MTMOS and TEOS).

El tratamiento de consolidación es el resultado de aplicar un protocolo específico $^{4}$, los conservadores necesitan conocer cómo, cuándo y bajo qué condiciones se puede aplicar un alcoxosilano como consolidante. El protocolo debe definirse a partir de una serie de aspectos relevantes como:

- El tipo de piedra y sus propiedades (componentes químicos mayoritarios, porosidad, tamaño de poro, absorción de agua, características mecánicas y dimensiones de la superficie a tratar.

- Características de los productos: naturaleza química del consolidante y disolventes y concentración utilizada. Cantidad de producto que debe ser aplicada y retenida por la piedra.

- Procedimiento de aplicación: proceso de aplicación, número de aplicaciones, intervalo de tiempo entre aplicaciones; condiciones ambientales requeridas durante y después del proceso de tratamiento y existencia de condiciones limitantes (temperatura).

La investigación en este campo se ha seguido desarrollando de forma activa en las últimas décadas, con el objetivo de resolver las dos grandes carencias que ofrecen los actuales consolidantes; su incapacidad para tratar rocas tipo calcita y la tendencia de los geles a fracturarse durante el proceso de secado.

La calcita por si misma contiene una cantidad muy pequeña de grupos hidroxilo en la superficie del mineral, necesarios para poder condensar con los alcoxisilanos. Weis y colaboradores ${ }^{5}$ han diseñado un tratamiento para generar estos grupos hidróxilo en la superficie del mineral por tratamiento con hidrogenotartrato de amonio en disolución a un determinado valor de $\mathrm{pH}$, una fórmula comercial Ilamada HCT. El hidrogenotartrato de amonio reacciona con la calcita

4 A.P. Ferreira Pinto, J. Delgado Rodrigues. Stone consolidation: The role of treatment procedures. Journal of Cultural Heritage 9 (2008) 38-53.

5 N. Weiss, I. Slavid, G. Wheeler, Development and assessment of a conversion treatment for calcareous stone. In: Vasco Fassina, Editor, Proceedings of the Ninth International Congress on Deterioration and Conservation of Stone, Venice. (2000), pp. 533-540. 
para formar tartrato de calcio (parte del mineral se consume en el proceso). El grupo funcional generado es capaz de condensar con los alcoxisilanos para formar nuevos enlaces C-O-Si que anclan el gel a la piedra (Fig. 5). En teoría, podrían hidrolizarse y liberar el gel, pero no todos los enlaces Si-O-C son comparables, a medida que el grupo orgánico enlazado al átomo de carbono aumenta su tamaño el impedimento estérico ejercido por este grupo voluminoso impide la ruptura por hidrolisis del enlace formado. El grupo tartrato es grande y aunque a nivel práctico el uso de esta fórmula es todavía limitado los estudios de laboratorio resultan muy prometedores.<smiles>C=CC(=O)C(O)C(O)C(=O)O[N]</smiles>

"hidrógeno tartrato de amonio"
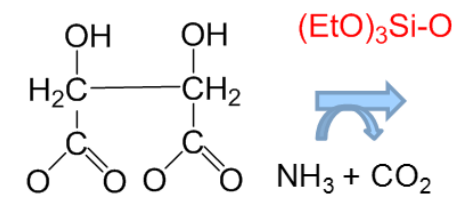

Calcita

Fig. 5. Uso de hidrogenotartrato y alcoxosilano como formula consolidante.

Una segunda aproximación consiste en utilizar compuestos alquil alcoxisilanos. Estos compuestos poseen una cadena alquílica con grupo alcoxido enlazados a un átomo central de silicio e hidrolizables y un cuarto sustituyente con un enlace $\mathrm{Si}-\mathrm{C}$ no hidrolizable (Fig. 6). El uso de estos compuestos mejora la fortaleza de las cerámicas obtenidas, al poseer dos funcionalidades y, además, una de ellas compatible con la superficie de la calcita ${ }^{6}$. Wheleer y colaboradores utilizan esta aproximación $n^{7}$. El sustrato inorgánico, la piedra calcita, es compatible con el grupo alquilo no hidrolizable. Mientras que los grupos alcoxisilano participan en el proceso de polimerización sol-gel. En el laboratorio se han observado mejoras muy significativas en el modulo de ruptura de las cerámicas obtenidas. Un ejemplo significativo de estos materiales alcoxisilanos híbridos o bifuncionales lo constituye el compuesto 2-(3,4-epoxiciclohexil)etil-trimetoxisilan (ECET) y GPDMS con aminopropiltrietoxisilano (APTES). El efecto del APTES en la reacción de apertura del anillo epóxido es muy diferente para ECET y GPDMS, ya que el anillo epóxido del ECET no está implicado en la adición de la amina y actúa, por tanto, como un modificador de red de la estructura híbrida resultante formada por grupos epoxiciclohexilo y aminopropilo enlazados covalentemente a la red de sílice.

\footnotetext{
6 E. Kyung Kima, J. Won, J-Y. Dob, S. D. Kim, Y. S. Kang. Effects of silica nanoparticle and GPTMS addition on TEOS-based stone consolidants. Journal of Cultural Heritage 10 (2009) 214-221.

7 G. Wheeler, J. Mendes-Vivar, E.S. Goins and C.J. Brinker. Evaluation of alkoxysilane coupling agents in the consolidation of limestone. In: Vasco Fassina, Editor, Proceedings of the Ninth International Congress on Deterioration and Conservation of Stone (Venice). Vol. 2 (2000), pp. 541-545.
} 


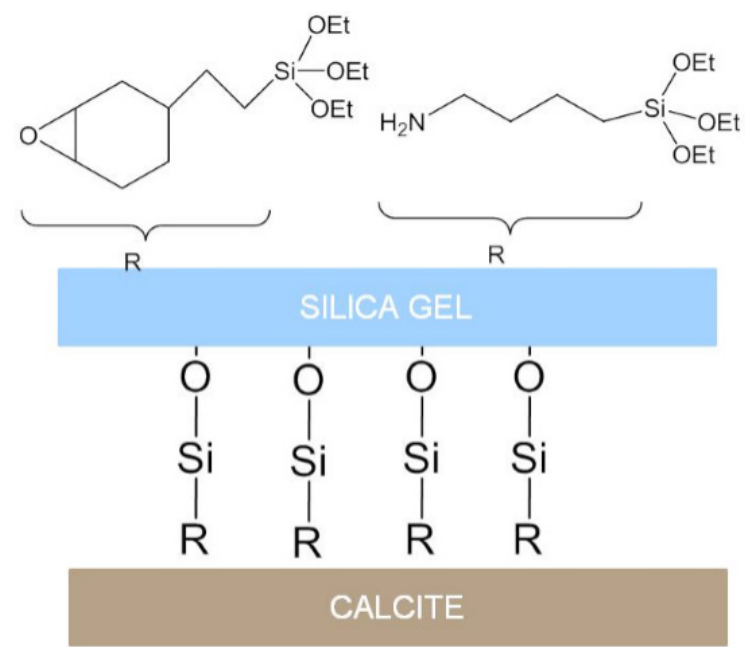

Fig. 6. Uso de alquil alcoxilanos como formula consolidante de piedra calcita.

La estrategia de los laboratorios Sandia ${ }^{8}$ consiste en utilizar un compuesto bifuncional capaz de enlazarse de forma selectiva a la superficie de la calcita y simultáneamente poseer grupos alcoxosilano capaces de polimerizar para formar la capa consolidante (Fig. 7). Estos alcoxisilanos bifuncionales se hidrolizan y polimerizan con la humedad atmosférica a la vez que pasivan la superficie de la calcita. A la vez que reaccionan con el agua adsorbida con la superficie, la posibilidad de enlazar grupos alquilo al átomo de silicio hace aumentar la hidrofobicidad del gel formado. Los enlaces formados son estables frente a la radiación ultravioleta.

Una aproximación para resolver el problema de fractura de estos materiales durante el proceso de secado es convertir el gel en un material más "manejable" introduciendo un segmento lineal tipo siloxano que sirva de puente entre dos zonas con estructura tridimensional dentro del gel (polidimetilsiloxano, PDMS) (Fig. 8). En este caso el gel y la piedra consolidada son menos frágiles y la transición entre piedra consolidada y piedra sin deteriorar menos abrupta. Esta idea se ha incorporado ya en las formulas comerciales ${ }^{9},{ }^{10}$. La adición de PDMS con cadenas flexibles e hidroxiapatita que genera una superficie más dura, reduce el

8 C. Jeffrey Brinker, Carol S. Ashley, Alan S. Seliinger, Randall T. Cygan, Kathryn L. Nagy, Roger Assink, Todd Alam, Sudeep Rao, S. Prabakar, Cathy S. Scotto. Sol-Gel Preservation of Mankind's Cultural Heritage in Objects Constructed of Stone. Sandia Report, SAND984251 UC-704 (1998).

9 R. Zárraga, J. Cervantes, C. Salazar-Hernández, G. Wheeler. Effect of the addition of hydroxyl-terminated polydimethylsiloxane to TEOS-based stone consolidants. Journal of Cultural Heritage 11 (2010) 138-144.

10 C. Salazar-Hernández, J. Cervantes, M.J. Puy-Alquiza, R. Miranda. Conservation of building materials of historic monuments using a hybrid formulation. Journal of Cultural Heritage 16 (2015) 185-19. 
riesgo de ruptura del gel durante la etapa de secado y aumenta la hidrofobicidad del gel. La hidroxiapatita mejora la resistencia al desgaste frente a factores medioambientales ${ }^{11}$.

Una importante línea de investigación es el diseño de nuevos procesos sol-gel utilizando alcoxisilanos como monómeros precursores para sintetizar nanomateriales con menores problemas de fractura ${ }^{12},{ }^{13},{ }^{14}$.

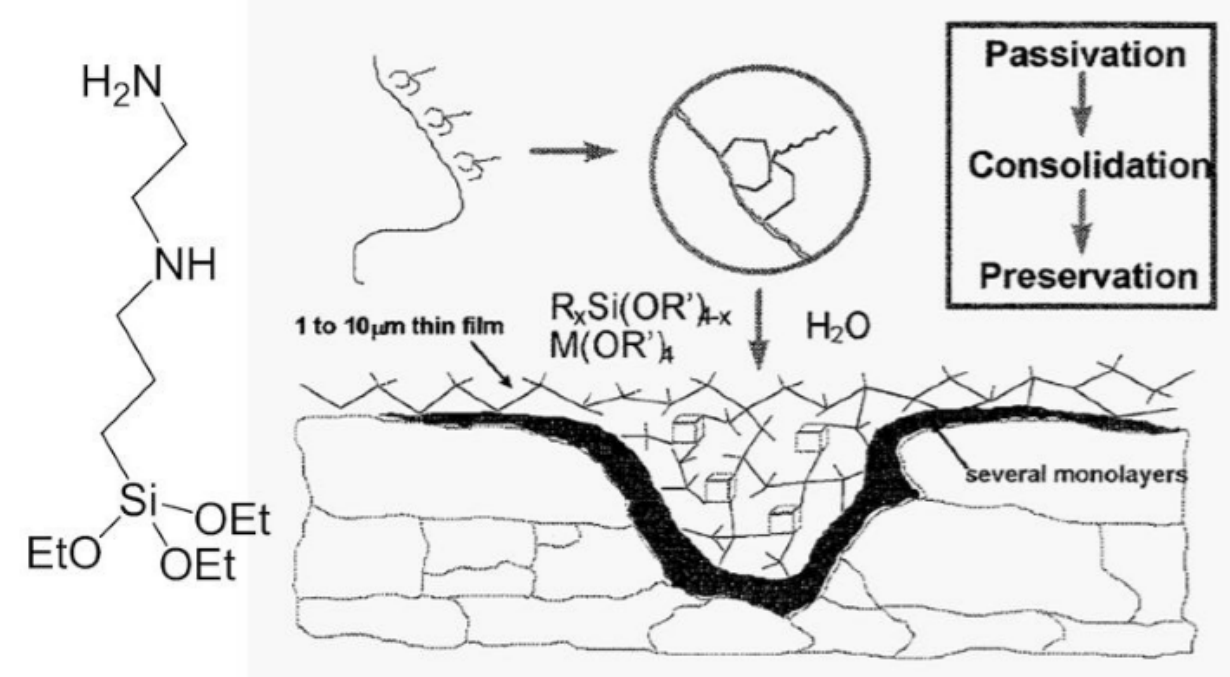

Fig. 7. Uso de alquil alcoxilanos bifuncionales como formula consolidante de piedra calcita.

El número creciente de esculturas del siglo XX y XXI que empiezan a presentar patologías similares al patrimonio tradicional ha puesto de manifiesto la necesidad de estudiar los procesos de degradación de las mismas y buscar soluciones a su conservación. La durabilidad que se ha presupuesto para estructuras de cemento Portland ha resultado no ser tal en determinadas ocasiones, bien debido a procesos intrínsecos asociados a su fabricación, bien a factores extrínsecos por su localización y mantenimiento. Estudios recientes de Blanco Varela y colaborado-

11 Y. Luo, L. Xiao, X, Zhang. Characterization of TEOS/PDMS/HA nanocomposites for application as consolitan/hydrophobic products on sandstones. Journal of Cultural Heritage (2014) http://dx.doi. org/10.1016/j.culher.2014.08.002.

12 J.F. Illescas, M.J. Mosquera. Surfactant-Synthesized PDMS/Silica Nanomaterials Improve Robustness and Stain Resistance of Carbonate Stone. Journal of physical chemistry C 115 (2011), 1462414634.

13 M.J. Mosquera, D.M. de los Santos, T. Rivas. Surfactant-Synthesized Ormosils with application to Stone Restoration. Langmuir, 26(9) (2010), 6737-674.

14 M.J. Mosquera, D. M. de los Santos, A. Montes, L. Valdez-Castro. New Nanomaterials for Consolidating Stone. Langmuir, 24 (2008) 2772-2778. 
res ${ }^{15}$ han demostrado la utilidad de los alcoxisilanos como consolidantes de este tipo de materiales. La consolidación es el resultado de la reacción de TEOS y las fases hidratadas del cemento portlandita $\mathrm{Ca}(\mathrm{OH})_{2}$ y gel $\mathrm{C}-\mathrm{S}-\mathrm{H}$.<smiles>C[Si]1(C)O[Si](C)(C)O[Si](C)(C)O[Si](C)(C)O[Si]2(O[Si](C)(C)O[Si](C)(C)O[Si](C)(C)O[Si]34O[Si](C)(C)O[Si](C)(C)O[Si](C)(C)O[Si](C)(O[Si](C)(C)O[Si](C)(C)O3)O4)O[Si](C)(O[Si](C)(C)O[Si](C)(C)O1)O2</smiles>

Fig. 8. Preparación de geles más flexibles mediante el uso de cadenas polidimetilsiloxano.

15 A.M. Barberena-Fernández, P.M. Carmona-Quiroga, M.T. Blanco-Varela. Interaction of TEOS with cementitious materials. Chemical and physical effects. Cement \& Concrete Composites 55 (2015) 145-152 